\title{
Estrutura organizacional e de coordenação pública na Amazônia: o caso de uma secretaria de saúde
}

O objetivo deste trabalho é analisar as relações organizacionais, a estrutura organizacional e as implicações para a coordenação dos serviços de saúde em um município paraense. Foram realizadas entrevistas com servidores e levantadas informações no website da prefeitura acerca da Secretaria de Saúde para subsidiar as discussões. Os resultados apontaram que a secretaria apresenta fragilidades na estrutura organizacional e na de coordenação, o que gera ineficiência. Sugeriuse uma nova estrutura organizacional para melhor orientação das ações organizacionais a vista de possivelmente melhorar a coordenação dos serviços de saúde prestado pela secretaria. Constata-se que as fragilidades organizacionais causam ineficiência, clamando por mais atenção do meio acadêmico e da administração pública sobre o impacto da estrutura e a coordenação no desempenho do serviço público.

Palavras-chave: Administração Públicas; Políticas Públicas; Eficiência; Serviços Públicos.

\section{Organizational and public coordination structure in the Amazon: the case of a health secretariat}

The objective of this work is to analyze the organizational relationships, the organizational structure and the implications for the coordination of health services in a municipality in Pará. They were held and interviews with servers and raised information on the town hall web site about the health department to support the discussions. The results showed that the secretariat has weaknesses in the organizational structure and in the coordination structure, which generates inefficiency. A new organizational structure was suggested to better orient organizational actions in order to possibly improve the coordination of health services provided by the secretariat. Notes is that organizational weaknesses cause inefficiency calling for more attention from academia and public administration on the impact of the structure and coordination in the performance of public service.

Keywords: Public Administration; Public Policies; Efficiency; Public Services.

Topic: Gestão Pública

Reviewed anonymously in the process of blind peer.
Received: 07/01/2021

Approved: 22/03/2021
Jakline Oliveira da Costa (iD)

Universidade Federal do Oeste do Pará, Brasil http://lattes.cnpq.br/1609235130952710 http://orcid.org/0000-0003-3860-8524

jaklineoliveira2@gmail.com

Erika Patricia de Sousa Araújo (iD

Universidade Federal do Oeste do Pará, Brasil

http://lattes.cnpq.br/1470732488226796

http://orcid.org/0000-0003-0022-9115

erikapatriciasousaaraujo1@gmail.com

Marco Aurélio Oliveira dos Santos (iD

Universidade Federal do Oeste do Pará, Brasil

http://lattes.cnpq.br/5333754444497512

http://orcid.org/0000-0003-3039-6264

marcoolivera santos@yahoo.com.br

\author{
Léo César Parente de Almeida \\ Universidade Federal do Oeste do Pará, Brasil \\ http://lattes.cnpq.br/4081029452825767 \\ http://orcid.org/0000-0003-1535-5325 \\ leocesarparente@gmail.com
}

Referencing this:

COSTA, J. O.; ARAÚJO, E. P. S.; SANTOS, M. A. O.; ALMEIDA, L. C. P.. Estrutura organizacional e de coordenação pública na Amazônia: o caso de uma secretaria de saúde. Revista Brasileira de Administração Científica, v.12, n.1, p.256-264, 2021. DOI: http://doi.org/10.6008/CBPC2179-684X.2021.001.0021 


\section{INTRODUÇÃO}

As organizações são agrupamentos de pessoas que buscam atingir objetivos ou superar problemas que sozinhos não teriam condições. Uma vez estruturadas, as organizações passam a enfrentar forças poderosas que as levam a se tornarem mais similares umas às outras (DIMAGGIO et al., 1983). Dentre estas foças, as políticas públicas representam um conjunto de ações formais que forçam organizações públicas a atuarem de forma homogênea.

As organizações públicas são as responsáveis por grande parte da produção e implementação das políticas públicas pensadas para resolver problemas nas áreas de educação, de segurança pública, de saneamento básico, de transporte entre outras ações governamentais que orientam as organizações públicas a atuar de forma isomórfica (DIMAGGIO et al., 1983). Assim, as políticas públicas, no papel de agente de isomorfismo organizacional, buscam minimizar ambiguidades e incertezas para assegurar previsibilidade na prestação de serviços e entrega de bens públicos.

Neste sentido, a necessidade de assegurar previsibilidade nas ações organizacionais subjaz a existência de uma estrutura de coordenação capaz de assegurar a eficiência nas atividades coletivas (CUNHA, 1999; SANTOS et al., 2011). Em razão disso, a coordenação é um processo de interação, de cooperação, de competição e de conflito entre indivíduos que estão interagindo e precisam de regularidades ou regras coletivamente aceitas para orientar as ações (MANTZAVINOS et al., 2004; WILLIAMSON, 1996). Neste processo, há um ator líder orientando a estrutura de coordenação segundo seus interesses, fato que, para Acemoglu (2005; 2008), Bachrach et al. (2011), North (1991a) e Woo et al. (2015), torna o ambiente organizacional um lócus de luta e de estratégias políticas de atores insurgentes que usam do seu poder para influenciar as decisões em prol de interesses particulares.

Nesta perspectiva, o ambiente organizacional é marcado por um conjunto de relações, de estratégias, de jogos de interesses e de poder que influenciam diretamente a eficiência organizacional. Desta forma, a estrutura organizacional é o mecanismo que deixa evidente a estrutura de autoridade, de responsabilidade, de especialização, de comunicação de relacionamento entre os diferentes atores e grupos de interesses que geram falhas no sistema de coordenação. Como a coordenação tem sido apontada como o Santo Graal na administração pública (EVANS et al., 1999; FUKUYAMA, 2013; PETERS, 2015), as organizações públicas precisam de uma estrutura bem delineada para assegurar a coordenação, boas relações intraorganizacionais, interorganizacionais e eficiência na prestação dos serviços públicos.

Segundo Cunha (1999), a estrutura organizacional compreende, fundamentalmente, o modo como a organização estabelece a estrutura de autoridade, divide o trabalho em múltiplas tarefas e a forma como estabelece a coordenação entre elas. Em vista disso, a estrutura organizacional deve ser delineada a partir dos objetivos organizacionais de modo a assegurar a clara atribuição das tarefas, as relações de autoridade e os mecanismos de coordenação das atividades buscando a máxima eficiência (CUNHA, 1999; JONES, 2010). Portanto, a estrutura organizacional ajuda a delinear um conjunto de normas que asseguram previsibilidade e similaridade nas ações das organizações públicas em diferentes contextos. 
Entretanto, como o contexto é marcado por distintas construções sociais, estratégias de re-scaling e lutas políticas de sujeitos insurgentes (ACEMOGLU, 2005; 2008), as organizações públicas tendem a apresentar diferentes padrões de desempenho. Por este motivo, contextos como o amazônico, por ser rico em recursos estratégicos para diversos setores econômicos, apresenta-se como palco de diferentes conflitos por terras e territoriais, lutas políticas e estratégias que impactam, diretamente, nas ações das organizações públicas (BECKER, 2014; HERRERA et al., 2013; KOHLHEPP, 2002; MELLO, 2006). Talvez seja por isso que, historicamente, os recursos amazônicos têm sido incorporados de forma irracional ao sistema produtivo e têm contribuído pouco com a promoção do desenvolvimento humano na região.

Um olhar mais crítico sobre as condições humanas da região não deixa dúvidas sobre o distanciamento que a separa das demais regiões brasileiras. A região apresenta baixo indicador de desenvolvimento humano, média de 0,68 entre os municípios, bem como um conjunto de fatores, como acentuadas taxas de pobreza, média dos municípios, que é de 25,75 , de vulnerabilidade à pobreza de 46,82 e de mortalidade infantil de 18,45 , colocando em risco a vida humana. Apesar de ser uma região rica em recursos naturais parece que o Estado e as organizações públicas não têm conseguido converter o uso dos recursos em melhores indicadores de desenvolvimento, sobretudo no que tange à saúde na região amazônica.

Em se tratando da saúde, o que se tem observado é que os indicadores de desenvolvimento da saúde, médias entre os municípios da região, é de 0,65 , fato que por si só chama a atenção da comunidade acadêmica de Administração Pública. Em um cenário no qual o Coronavírus (COVID-19) figura como um dos piores problemas de saúde pública para o ano de 2020, não só pela escassez de tratamentos (LIPPI et al., 2020; MONAJJEMI et al., 2020), mas por colocar em evidência a capacidade do sistema público de saúde ser eficiente no atendimento à população, o que faz com que a capacidade de coordenação das organizações públicas possa representar risco à saúde, um limitante ao desenvolvimento humano e a qualidade de vida das comunidades amazônicas.

Especificamente, o Pará é o estado amazônico com um dos piores indicadores de desenvolvimento da saúde do Brasil, média de 0,60 entre os municípios. Nesse sentido, a comunidade de administração pública é instigada a questionar o porquê desta realidade. Espera-se que as organizações públicas sejam eficientes em promover o bem-estar social e entregar os serviços médicos de forma planejada, organizada, coordenada e controlada. Para tanto, estas organizações precisam ser projetadas para fornecer uma gama relativamente estreita de serviços, desenvolver perícia em uma área política e capacidade de coordenação para responder com eficiência aos desafios impostos pelo COVID-19 (ANNE, 2015; EVANS et al., 1999; FUKUYAMA, 2013; PETERS, 2015). Na falta desta capacidade de coordenação, as organizações optam por orientar um conjunto de crenças, valores e normas informais que representam uma ruptura com as regras formais construídas para assegurar a previsibilidade das ações organizacionais (NORTH, 1991á; 1991b). Portanto, tendem a apresentar diferentes padrões de desempenho.

Neste cenário, fragilidades no delineamento organizacional tendem a intensificar a ineficiência organizacional, pois não se deixa claro a estrutura de autoridade, de responsabilidade, de especialização, de 
comunicação de relacionamento entre outros elementos importantes para mitigar falhas no sistema de coordenação (EVANS, 1995; NORTH, 1991a; 1991b; SANTOS et al., 2011). Por consequência, fragilidades na estrutura organizacional e de coordenação das organizações públicas de saúde pode ser um agravante ao desenvolvimento dos serviços de saúde, especialmente nos municípios paraenses.

Como o município é um espaço político administrativo mais visível e mais próximo do cotidiano do cidadão (CASTRO, 2003; KLERING, 2009), fragilidades na estrutura organizacional e de coordenação podem levar a falhas na entrega dos serviços de saúde no estado do Pará. Assim, as organizações municipais de saúde precisam contar com uma estrutura organizacional e de coordenação eficiente para planejar, implementar, controlar e, sobretudo, para coordenar a entrega dos serviços de saúde à população (SILVA et al., 2010). Logo, fragilidades no designer organizacional por representar falhas na divisão de tarefas, na estrutura hierárquica, na especialização entre outros fatores que asseguram eficiência na coordenação dos serviços de saúde no Pará.

Desse modo, o artigo tem por objetivo analisar as relações organizacionais, a estrutura organizacional e as implicações para a coordenação e desenvolvimento do serviço de saúde pública em um município paraense. O trabalho é justificado por mostrar como o contexto e o ambiente interno das organizações públicas tem o poder de romper com o isomorfismo organizacional, minimizar a capacidade de coordenação e a eficiência na entrega dos serviços de saúde. Ademais, busca ressaltar a importância do designer organizacional para eficiência na gestão pública para melhor atendimento da população e deste para desenvolvimento acadêmico e social. Além desta introdução, o trabalho apresenta a metodologia, os resultados e discussões e as considerações finais.

\section{METODOLOGIA}

Este artigo representa um esforço exploratório para entender uma realidade e nortear a produção de novas pesquisas. Foi realizada na Secretaria Municipal de Saúde de Alenquer, pertencente a mesorregião do Baixo Amazonas paraense. Alenquer foi elevada à categoria de município em 10 de junho de 1881 . 0 município está situado a cerca de $696 \mathrm{Km}$ em linha reta da capital do Pará (Belém), tem aproximadamente 56480 habitantes, sendo que metade da população vivia abaixo da linha da pobreza em 2017. Neste cenário, a Secretaria Municipal de Saúde é a responsável por planejar, implementar e coordenar as políticas de saúde para toda a população.

É um órgão que se subdivide em níveis hierárquicos e que segue padrões administrativos, possuindo elementos organizacionais que orientam a eficiência. Para obter as informações, foram realizados levantamentos de informações no website da prefeitura e entrevistas abertas com servidores da secretaria com intuito de coletar informações que permitissem explorar e entender o problema, a partir da percepção dos indivíduos ou grupos em seu ambiente cotidiano (CRESWELL, 2010). As entrevistas foram analisadas seguindo os princípios da Análise de Conteúdo para buscar captar os significados e construir uma descrição sistemática dos resultados discutidos em seguida. 


\section{RESULTADOS E DISCUSSÃO}

A Secretaria Municipal de Saúde de Alenquer tem seis unidades de atendimento básico de saúde que se encontram em funcionamento. Cada unidade está sob responsabilidade de um coordenador específico dentro de um sistema hierárquico de decisão. Segundo a entrevistada $B$, a coordenação define o que será realizado e cabe às unidades acatar e planejar ações de forma autônoma. Neste sentido, a autonomia é um elemento complexo, pois depende de como o gestor emite mandatos para a burocracia e da qualidade da burocracia.

A qualidade da burocracia é intrínseca a cada um dos servidores de carreira. Constatou-se que todos os coordenadores apresentam graduação e alguns com pós-graduação em áreas diretamente relacionadas com as atividades fins da secretaria de saúde. Estes atores, ao apresentarem conhecimento real da área de atuação, estão aptos a inovar na prestação dos serviços de forma mais autônoma, pois, segundo Evans (1995) e Fukuyama (2013), quando uma agência está cheia de incompetentes é desejável ter regras claras para limitar sua discrição, ao passo que se a agência conta com profissionais com pós-graduação é prudente reduzir o limite das regras na esperança de incentivar um comportamento inovador. Além disso, como são atores qualificados, tendem a apresentar mais comprometimento com o trabalho (ANNE, 2015). Logo, a presença destes coordenadores qualificados favorece a redução de regras em prol da inovação.

Entretanto, conforme o entrevistado $D$, há um déficit de recursos humanos em todos os setores, fato que sobrecarrega os profissionais com atividades que às vezes vão além de suas funções. Esta carência de profissionais, para Peters (2015), força os servidores a ter concentração em atividades rotineiras que limitam a capacidade destes de vender ideias ou mesmo transmitir informações para os tomadores de decisões. Fukuyama (2013) também alega que este distanciamento entre o nível de rua e os tomadores de decisões cria fragilidades nas conexões e alinhamento entre as políticas, a realidade empírica e os resultados esperados pelo governo.

Para Parsons (2004), ao distanciar a informação da tomada de decisão, prejudica-se a capacidade de construção de laços e soluções mais acertadas pelos governos. Ademais, reduz a capacidade de inovação e imitação que ocorre em um grupo social que está a aprender coletivamente, ou seja, reduz-se a capacidade construção de novas crenças e valores, elementos que favorecem a cooperação e a coordenação inter e intraorganizacional (MANTZAVINOS et al., 2004). Assim, os gestores da secretaria precisam planejar, dirigir e controlar recursos financeiros, bem como construir e liderar equipes de trabalho em um ambiente de apoio mútuo buscando assegurar a cooperação e integração de atores em torno de objetivos comuns (BERTELLI, 2006), dentro de uma estrutura organizacional que deixa clara as relações de autoridade e responsabilidade.

Contudo, o que se pode observar pelas entrevistas é que, apesar de terem autonomia, os coordenadores não contam com uma estrutura física e organizacional adequada para a prática de muitas tarefas, o que causa desconforto para servidores e usuários. Assim sendo, o que se observa na secretaria é uma tentativa de favorecer a autonomia dos servidores sem uma estrutura adequada para descentralizar autoridade e responsabilidade. Consequentemente, a fragilidade no design organizacional limita a 
autonomia, pois não se permite delegar autoridade e responsabilidade enfraquecendo o processo de inovação e a coordenação das ações públicas da secretaria.

Para os entrevistados $B$ e $D$, é preciso organizar a unidade conforme as normas e procedimentos para expandir a atenção básica e colocar mais postos em funcionamento para melhor atendimento ao público. A presença desta falta de organização faz com que muitas pessoas nem sempre sigam as normas (Entrevistado $B$ e D). Fato que deixa claro que o ambiente organizacional em estudo é um lócus de luta e de estratégias políticas de atores orientados pelos seus interesses particulares (ACEMOGLU, 2005; 2008; BACHRACH et al., 2011; BECKER, 1983; WOO et al., 2015). Há diferentes modelos mentais que precisam ser alinhados para melhorar o desempenho do sistema de coordenação organizacional.

À medida que os atores se inter-relacionam, há um processo cumulativo de aprendizado que permite aos indivíduos dar forma aos fatos do ambiente no qual estão inseridos (MANTZAVINOS et al., 2004). E na presença desta fragilidade no delineamento organizacional que os atores passam a ser orientados por normas socialmente aceitas que direcionam as ações e decisões organizacionais. Por consequência, estas regras informais são determinantes na coordenação das ações organizacionais (NORTH, 1991ạ; 1991b). Portanto, estas fragilidades passam a explicar a ineficiência organizacional da secretaria.

Diante das fragilidades observadas, a estrutura organizacional da secretaria de saúde de Alenquer não é capaz de descrever de forma simplificada a estrutura de autoridade, de responsabilidade, de especialização e comunicação das ações, fazendo com que as ações se tornem desordenadas e ineficientes. Faz-se necessário um novo design organizacional para tornar as ações mais eficientes, pois para Cunha (1999), a estrutura é o esqueleto da organização de modo a influenciar e constranger o comportamento da e na organização.

Como observam Santos et al. (2011), as interações ocorrem entre os departamentos, os níveis e as posições de modo a gerar um processo de diferenciação organizacional. Em vista disso, uma nova estrutura permitirá definir tarefas, as relações de autoridade e de responsabilidade, bem como orientar o sistema de coordenação das ações ao agrupar ações e atores a partir de atividades homogêneas permitindo delinear normas de relacionamento e interação. Deste modo, as interações departamentais criam linhas de comunicação orientadas para os objetivos organizacionais e também possibilitam a construção de relacionamento informais.

Neste cenário, um novo delineamento organizacional favorece a identificação da amplitude de controle, a descentralização das atividades, a especialização, a formalização, integração e profissionalização (CARBONE, 2000; CUNHA, 1999; SILVA et al., 2010), de modo a melhor orientar o processo de autonomia ofertada pelo secretário aos coordenadores. Dentre os elementos descritos, o mais importante é a formalização, pois, tal como especificado por Cunha (1999), ela representa o quanto as atividades são definidas por escrito, por isso a possibilidade de o indivíduo realizar o seu trabalho à sua maneira. Com isso, a formalização da estrutura organizacional e das ações organizacionais rompe com a falta de seguimento das normas por parte dos servidores. 
Ao formalizar o design organizacional com cargos e tarefas, busca-se formalizar as ações para assegurar a previsibilidade das ações individuais dos atores enquanto ocupantes de um cargo. Da mesma forma que favorecem a especialização dos atores no cargo e o recolhimento e transmissão de informações internas minimizando a possibilidade de cada setor atuar de forma autônoma e por criar seus padrões e regras simbólicas que afetam a eficiência da organização. Deste modo, o organograma, Figura 1, representa uma proposta de departamentalização organizacional a partir das funções tencionando aumentar a eficiência e aprimorar as inter-relações internas da Secretaria de Saúde.

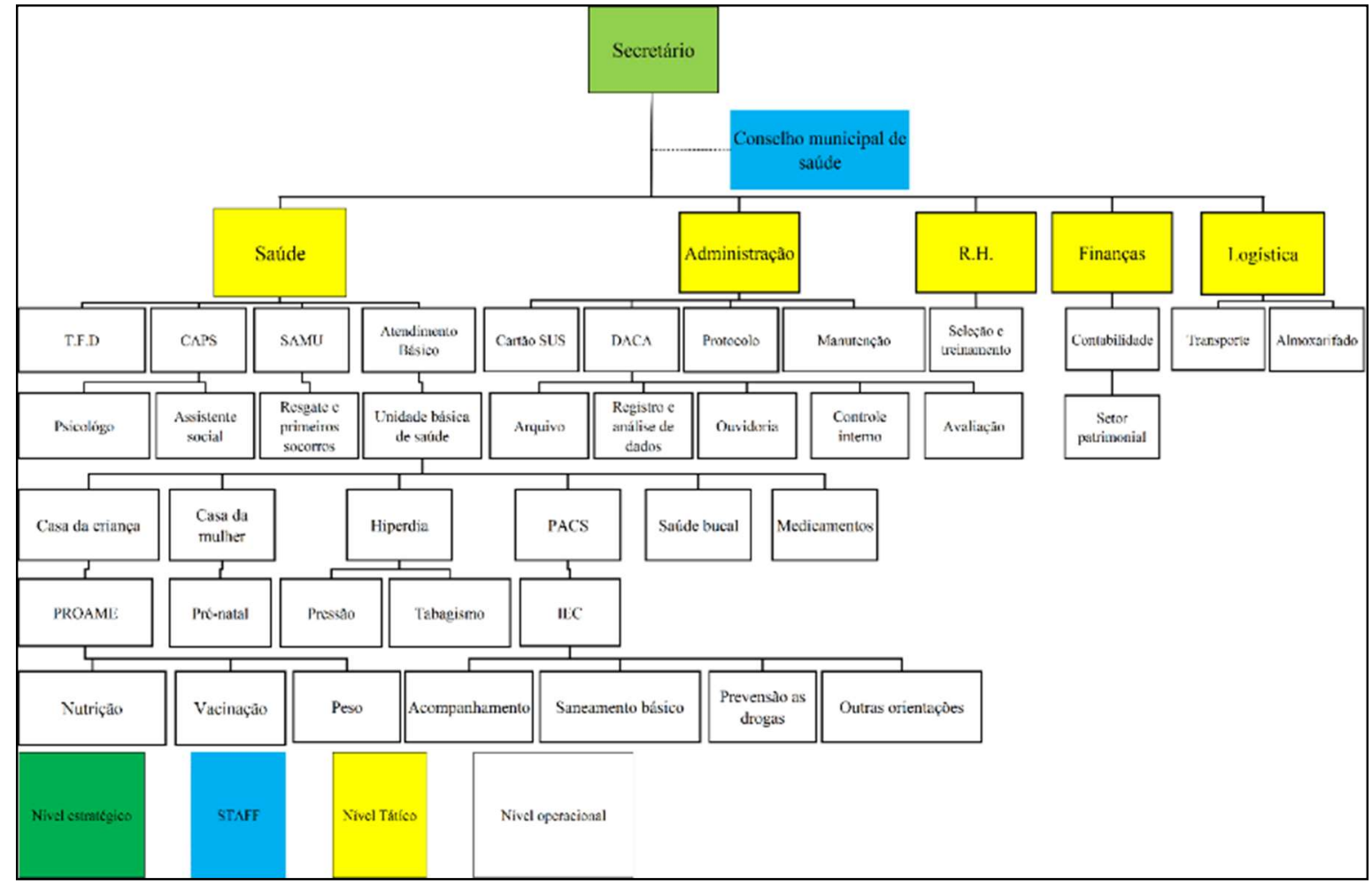

Figura 1: Proposta de departamentalização.

A proposta cria uma hierarquia de comando e divide a organização em cinco setores, saúde, administração, recursos humanos, finanças e logística, além de contar com a linha de staff. Neste sentido, o secretário estaria no nível estratégico, responsável pelo processo decisório e pelo planejamento, ao passo que os coordenadores estariam no nível tático e seriam os responsáveis por transformar as ações planejadas em estratégias práticas. Por fim, no nível operacional, as estratégias são colocadas em prática. Logo, a proposta de departamentalização cria níveis de comando estratégico, tático e operacional, define as linhas de comando, os departamentos, os cargos e os serviços da organização, permitindo ao gestor uma visão clara e simplista, facilitando o planejamento e o controle organizacional e a integração dos colaboradores aos objetivos da organização.

Espera-se que seja uma imagem que espelhe a estrutura e suas características, de modo a influenciar o comportamento dos atores internamente para melhoria e integração do sistema básico de saúde de modo a facilitar o diagnóstico e o tratamento das diversas enfermidades que assolam a sociedade a partir de uma estrutura adequada e necessária para atender a demanda de modo eficiente. 


\section{CONCLUSÕES}

Ao analisar a estrutura organizacional da secretaria municipal de saúde de Alenquer-PA foi possível observar que a ausência de uma estrutura física adequada, bem como o delineamento organizacional adequado dificulta a coordenação e a realização das atividades organizacionais. Apesar de possuir a maioria dos materiais para a realização de suas tarefas, ainda faz necessário a disponibilização de materiais eletrônicos e a criação de áreas que possam atender às necessidades tanto da execução dos serviços quanto dos colaboradores.

Assim, a secretaria precisa de uma a organização para melhorar os procedimentos administrativos, o planejamento, a direção, o controle e, sobretudo, a coordenação das atividades entre as unidades básicas de saúde. Isso é necessário por se tratar de um órgão da rede pública com várias ramificações nas ações em torno da saúde, portanto, precisa ser projetada para atender às necessidades dos usuários do serviço, de modo que possam atingir suas metas e objetivos.

A partir das informações coletadas, pode-se notar que a organização apresenta falhas administrativas. Além do mais, a fragilidade do desenho organizacional representa um fator que inibe a inovação das ações organizacionais pretendida pelo secretário ao conceder autonomia aos coordenadores setoriais. Do mesmo modo que a falta de formalização permite que os servidores optem por não seguir as regras, fatos que representam fontes de ineficiência na execução dos serviços realizados. Sendo assim, o estudo permitiu constatar que o funcionamento desta organização pública é limitado pela interação dos setores e indivíduos, ou seja, pelas relações intraorganizacionais.

Neste cenário, a organização é caracterizada por um conjunto de regras socialmente construídas e consolidadas pelo contexto social no qual a organização está inserida. Ou seja, um contexto marcado pela fragilidade social e organizacional. Decerto que o estudo possibilitou melhor entendimento sobre estes elementos, agregando informações sobre práticas de gestão que levam até a ineficiência de um órgão público, de modo a contribuir com o meio acadêmico e para a administração pública sobre o entendimento o impacto das relações intraorganizacionais no serviço público.

No entanto, por se tratar de um contexto específico, novas pesquisas comparativas precisam ser realizadas para entender como as relações intraorganizacionais, os processos de coordenação internos e se os municípios circunvizinhos seguem os processos institucionais e diretrizes organizacionais de modo a auxiliar outras pesquisas no âmbito da saúde pública no contexto amazônico.

\section{REFERÊNCIAS}

ACEMOGLU, D.. Politics and economics in weak and strong states. Journal of Monetary Economics, v.52, n.7, p.11991226, 2005

ACEMOGLU, D.. Oligarchic versus democratic societies. Journal of the European Economic Association, v.6, p.1-44, 2008.

ANNE, T.. The dilemmas of organisational capacity. Policy and Society, v.34, n.3-4, p.209-217, 2015.
BACHRACH, P.; BARATZ, M. S.. Duas faces do poder. Revista de Sociologia e Política, v.19, n.40, p.149, 2011.

BECKER, B. K.. O uso político do território: questões a partir de uma visão do terceiro mundo. In: BECKER, B. K.; COSTA, R. H.; SILVEIRA, C. B.. Abordagens políticas da espacialidade. Rio de Janeiro: UFRJ, 1983.

BECKER, B. K.. A Amazônia como um território estratégico e 
os desafios às políticas públicas. In: SIFFERT FILHO, N. F.; SANTIAGO, M. C.; MAGALHÃES, W. A.; LASTRES, H. M. M.. Um olhar territorial para o desenvolvimento. Rio de Janeiro: Banco Nacional de Desenvolvimento Econômico e Social, 2014. p.396-401.

BERTELLI, A. M.. Determinants of Bureaucratic Turnover Intention: Evidence from the Department of the Treasury. Journal of Public Administration Research and Theory, v.17, n.2, p.235-258, 2006.

CARBONE, P. P.. Cultura organizacional do setor público brasileiro: desenvolvendo uma metodologia de gerenciamento da cultura. Revista de Administração Pública, v.34, n.2, p.1-5, 2000.

CASTRO, I. E.. Instituições e território. Possibilidades e limites ao exercício da cidadania. Geosul, v.18, n.36, p.7-28, 2003.

CRESWELL, J. W.. Projeto de pesquisa: métodos qualitativos, quantitativos e mistos. 3 ed. Porto Alegre: Artmed, 2010.

CUNHA, M. P.. Estrutura organizacional: dimensões, determinantes e formatos. In: CUNHA, M. P.. Teoria organizacional: perspectivas e prospectivas. Lisboa: Dom Quixote, 1999. p.251-279.

DIMAGGIO, P.; POWELL, W.. The Iron Cage Revisited: Institutional Isomorphism and Collective Rationality in Organizational Fields. American Sociological Review, v.48, n.2, p.147-160, 1983.

EVANS, P.. Embedded autonomy: states and industrial transformation. New Jerse: Princeton University Press, 1995.

EVANS, P.; RAUCH, J. E.. Bureaucracy and Growth: A CrossNational Analysis of the Effects of "Weberian" State Structures on Economic Growth. American Sociological Review, v.64, n.5, p.748, 1999.

FUKUYAMA, F.. What Is Governance?. Governance, v.26, n.3, p.347-368, 2013.

HERRERA, J. A.; MOREIRA, R. P.. Resistência e conflitos sociais na amazônia: a luta contra o empreendimento Hidrelétrico de Belo Monte. Campo - Território: Revista De Geografia Agrária, p.130-151, 2013.

JONES, G. R.. Teoria das organizações. São Paulo: Pearson, 2010.

KLERING, L. R.. Políticas e estratégias públicas municipais: novos desafios e perspectivas para o desenvolvimento local.
Redes, v.13, n.2, p.144-160, 2009.

KOHLHEPP, G.. Conflitos de interesse no ordenamento territorial da Amazônia brasileira. Estudos Avançados, v.16, n.45, p.37-61, 2002.

LIPPI, G.; PLEBANI, M.. Procalcitonin in patients with severe coronavirus disease 2019 (COVID-19): A meta-analysis. Clinica Chimica Acta, v.505, p.190-191, 2020.

MANTZAVINOS, C.; NORTH, D. C.; SHARIQ, S.. Learning, Institutions, and Economic Performance. Perspectives on Politics, Cambridge, v.2, n.01, p.75-84, 2004.

MELLO, N. A.. Políticas territoriais na Amazônia. São Paulo: Annablume, 2006

MONAJJEMI, M.; MOLLAAMIN, F.; SHOJAEI, S.. An overview on Coronaviruses family from past to Covid-19: introduce some inhibitors as antiviruses from Gillan's plants. Biointerface Research in Applied Chemistry, v.10, n.3, p.5575-5585, 2020.

NORTH, D. C.. Institutions. Journal of Economic Perspectives, Berkeley, v.5, n.1, p.97-112, 1991a.

NORTH, D. C.. Institutions, ideology, and economic performance. Cato J., v.11, p.477, 1991b.

PARSONS, W.. Not Just Steering but Weaving: Relevant Knowledge and the Craft of Building Policy Capacity and Coherence. Australian Journal of Public Administration v.63, n.1, p.43-57, 2004.

PETERS, B. G.. Policy capacity in public administration. Policy and Society, v.34, n.3-4, p.219-228, 2015.

SANTOS, L. G. A.; ROSSONI, L.; SILVA, C. L. M.. Condicionantes estruturais dos relacionamentos intraorganizacionais: uma análise da influência sobre relações de comunicação e decisão. Revista de Administração Mackenzie, v.12, n.1, p.139-168, 2011.

SILVA, C. L. M.; FONSECA, V. S.. Estruturação da estrutura organizacional: o caso de uma empresa familiar. Revista de Administração Contemporânea, v.14, p.11-32, 2010.

WILLIAMSON, O. E.. The Mechanisms of Governance. New York: Oxford University Press., 1996.

WOO, J. J.; RAMESH, M.; HOWLETT, M.. Legitimation capacity: System-level resources and political skills in public policy. Policy and Society, v.34, n.3-4, p.271-283, 2015.

A CBPC - Companhia Brasileira de Produção Científica (CNPJ: 11.221.422/0001-03) detém os direitos materiais desta publicação. Os direitos referem-se à publicação do trabalho em qualquer parte do mundo, incluindo os direitos às renovações, expansões e disseminações da contribuição, bem como outros direitos subsidiários. Todos os trabalhos publicados eletronicamente poderão posteriormente ser publicados em coletâneas impressas sob coordenação da Sustenere Publishing, da Companhia Brasileira de Produção Científica e seus parceiros autorizados. Os (as) autores (as) preservam os direitos autorais, mas não têm permissão para a publicação da contribuição em outro meio, impresso ou digital, em português ou em tradução. 